\title{
Research of Planetary Gear Fault Diagnosis Based on Multi-Scale Fractal Box Dimension of CEEMD and ELM
}

\author{
Xihui Chen - Gang Cheng* - Hongyu Li - Yong Li \\ China University of Mining and Technology, School of Mechatronic Engineering, China
}

The planetary gear is the most critical part of a drive transmission system, and its faults will affect the reliability of the equipment, and even cause accidents. Therefore, it is of great significance to study the fault diagnosis of the planetary gear. A method of planetary gear fault diagnosis based on the multi-scale fractal box dimension of complementary ensemble empirical mode decomposition (CEEMD) and extreme learning machine (ELM) is proposed. The original vibration signal is decomposed by CEEMD, and a series of intrinsic mode functions (IMFs) are obtained. Some effective IMFs are extracted, and their reconstructed signal associated with the feature information is obtained. The reconstructed signal is analysed with multi-scale analysis, and the fault feature information contained in the signals with different scales is quantified and extracted via a fractal box dimension. The status recognition of planetary gear is achieved by combining ELM. The experiments show that the proposed method is effective at diagnosing planetary gear faults.

Keywords: Fault diagnosis, Planetary gear, CEEMD, Multi-scale, Fractal box dimension, ELM

Highlights

- A method of planetary gear fault diagnosis based on the multi-scale fractal box dimensions of CEEMD and ELM is proposed.

- The effective IMFs which associate with the shock feature information are selected by the kurtosis criterion.

- The multi-scale fractal box dimension of the reconstructed signal is defined as the fault feature information.

- The status recognition of a planetary gear is achieved by combining ELM.

\section{O INTRODUCTION}

The planetary gear is widely used in all kinds of drive transmissions of large electromechanical equipment, and it is the most critical part of a drive transmission system. A fault in a planetary gear will affect the reliability of the equipment, and even causes accidents [1]. Therefore, to study planetary gear fault diagnosis has great significance. The complex structure of planetary gear and the changes of working parameters, such as speed and load, will cause the vibration signal of planetary gear to be non-stationary, and it also has the characteristics of frequency modulation (FM) and amplitude modulation (AM) [2]. Therefore, the traditional time-domain analysis and frequencydomain analysis that only have statistical properties and global significance are not suitable for processing the non-stationary signals; the feature extraction method for non-stationary signals and the advanced classification method should be studied.

In order to obtain the useful information generated by planetary gears, some time-frequency analysis methods are proposed. The time-frequency analysis method which has the best application effect is empirical mode decomposition (EMD) [3] proposed by Huang et al. [4]. A series of IMFs with strict definitions can be obtained from the original vibration signal [5]. However, EMD has a major drawback: modal aliasing. To solve this problem, EEMD is proposed [6]. The Gaussian white noise is added to the original signal to change the extreme points, and an IMF set can be obtained by EMD. The Gaussian white noise is added multiple times, and a series of IMF sets can be obtained. The final IMFs can be obtained by the average of a series of IMF sets, and the added Gaussian white noise is eliminated according to its uniform characteristics. However, due to the added Gaussian white noise is eliminated by ensemble average; the reconstructed signal of the IMFs still contains residual noise. CEEMD is the latest method to improve the shortcomings of EMD; it can achieve a better decomposition effect which is beneficial for extracting the features [7]. A pair of Gaussian white noises with opposite symbols are respectively added to the original vibration signal, following the EMD process is carried out. CEEMD can solve the problems of modal aliasing and energy leakage, and the problem of reconstruction error is alleviated by using complementary pairs of Gaussian white noise.

The collected vibration signal near the planetary gear also contains the vibration produced by other transmission components. With CEEMD, a large part of vibration interference produced by other transmission components and the feature information produced by planetary gears are divided into different IMFs. Only some IMFs associated with the feature information generated by planetary gear faults and some IMFs do not contain the information that we 
need. The effective IMFs, including the most relevant, feature information for recognizing planetary gears should be selected. Next, it is necessary to extract the fault feature information. The multi-scale algorithm has the advantages of high efficiency, good convergence, and high precision, and it can show the features of the signals with different scales [8]. The fractal dimension is an important parameter in the quantitative description of nonlinear behaviour, and it mainly includes the Hausdorff dimension, similarity dimension and box dimension, etc. Among them, the fractal box dimension is widely used [9]. The fractal box dimension establishes the relationship with the nonlinear problem based on the box-filling idea. For the status change of a mechanical transmission system, it can be used to describe the statistical self-similarity feature of the fractal boundary of the vibration signal. Thus, the multi-scale fractal box dimension combining multi-scale analysis and fractal box dimension can be used to extract the fault feature information.

Fault classification is necessary after extracting the fault feature information; some classification methods such as support vector machine (SVM), ELM, and other neural networks are proposed [10]. SVM is a data classification method that is suitable for processing small samples, and the nonlinear mapping of the date is realized by the kernel function. Therefore, the kernel function and its parameters are crucial. With the development of optimization algorithms, some parameter optimization algorithms such as artificial colony bee algorithm and genetic algorithm are combined with SVM [11]. However, that will increase the complexity and computational burden. The neural network has developed into an effective classification method in fault diagnosis, but the traditional algorithms have several shortcomings, such as slow training speed, the fact that they easily fall into a local minimum point and sensitive learning rate. ELM is a new learning algorithm of the singlehidden layer feed-forward neural network, its weights between the input layer and hidden layer and the threshold of hidden neurons are randomly generated, and there is no need to adjust them in the training process. Only the number of hidden neurons needs to be set, and the unique optimal solution can be obtained [12]. ELM has the advantages of easy parameter selection, fast learning speed, and good generalization performance, and it can be applied in the classification of planetary gear status.

This paper is structured as follows. In Section 1, a mathematical model of planetary gear fault diagnosis based on a multi-scale fractal box dimension of CEEMD and ELM is built. In Section
2, the experiment equipment of planetary gear fault simulation is introduced. In Section 3 and Section 4, the vibration signals are processed by the proposed method, and its effectiveness and applicability are verified. In the last section, many conclusions are obtained.

\section{MODEL BUILDING}

\subsection{CEEMD}

CEEMD is an improved algorithm based on EMD; a pair of Gaussian white noises with opposite symbols are added to the original signal, and EMD and the ensemble average process are conducted. That can eliminate the residual noise contained in the reconstructed signal and improve the completeness of the decomposition process [13]. CEEMD can be expressed as follows:

Step 1: The original vibration signal is $x(t)$, and $s$ pairs of Gaussian white noises with opposite symbols are added, and a $2 s$ added white noise signal can be obtained.

$$
\left[\begin{array}{l}
x_{s+}(t) \\
x_{s-}(t)
\end{array}\right]=\left[\begin{array}{cc}
1 & 1 \\
1 & -1
\end{array}\right]\left[\begin{array}{l}
x(t) \\
n_{s}(t)
\end{array}\right],
$$

where $n_{s}(t)$ is the $s^{\text {th }}$ added Gaussian white noise. $x_{s+}(t)$ is the signal added to the $s^{\text {th }}$ Gaussian white noise with a positive symbol, and $x_{s-}(t)$ is the signal added to the $s^{\text {th }}$ Gaussian white noise with a negative symbol.

Step 2: For the signal-added Gaussian white noise, EMD [4] is carried out, respectively. $2 s$ sets of IMFs are obtained, and they are expressed as follows:

$$
\begin{aligned}
& \left.\left[\begin{array}{l}
i m f_{s+}^{i}(t) \\
i m f_{s-}^{i}(t)
\end{array}\right]=E\left(\begin{array}{cc}
1 & 1 \\
1 & -1
\end{array}\right]\left[\begin{array}{l}
x(t) \\
n_{s}(t)
\end{array}\right]\right), \\
& s=1,2, \ldots, S ; \quad i=1,2, \ldots, I,
\end{aligned}
$$

where $E$ ( ) represent EMD process, $S$ is the number of the added Gaussian white noise, and $I$ is the number of the obtained IMFs.

Step 3: The process of ensemble average is processed, and the final IMFs can be obtained; they can be expressed as follows:

$$
I M F_{i}=\frac{1}{2 S} \sum_{s=1}^{S}\left(i m f_{s+}^{i}(t)+i m f_{s-}^{i}(t)\right) .
$$

Step 4: The vibration signal can be expressed as follows:

$$
x(t)=\sum_{i=1}^{I} I M F_{i}(t)+r(t)
$$

where $r(t)$ is the residual signal. 


\subsection{Extraction of Effective IMFs}

The signal collected by the vibration sensor installed near the planetary gear also contains the vibration produced by other transmission components, which is reduced significantly in the vibration transfer process, and they have a certain difference with the vibration produced by the monitored planetary gear. With CEEMD, a large part of vibration interference produced by other transmission components and the feature information produced by planetary gear are divided into different IMFs. Only some IMFs associate with the feature information produced by planetary gear faults, and the effective IMFs associating with the fault feature information produced by the planetary gear should be selected. The shock components are generated during the meshing process of the planetary gear, and they have different changes when different faults of the planetary gear occur, so the fault feature information is usually contained in the shock components generated during the meshing process. Kurtosis is strongly related to the shock components, so the effective IMFs associated with feature information can be selected by kurtosis. Assuming $I M F_{i}=\left(x_{1}, x_{2}, \ldots, x_{N}\right)$, The definition of kurtosis is as follows [14]:

$$
K=\frac{\sum_{n=1}^{N}\left(\left|x_{n}\right|-\bar{x}\right)^{4}}{\sigma^{4}},
$$

where $\bar{x}$ is the mean value and $\sigma$ is the standard deviation of the signal.

\subsection{Multi-Scale Fractal Box Dimension}

The effective IMFs including the fault shock components are selected, and their reconstructed signal contains the main fault feature information generated by planetary gear fault. Next, the fault feature extraction and quantization of the reconstructed signal should be analysed.

\subsubsection{Multi-Scale Analysis}

Multi-scale analysis can show the features of the signals with different scales. For a time-domain signal with length $H ; x_{1}, x_{2}, \ldots, x_{H}$, it is divided into several time series with different lengths according to the scale factor $\tau$. The average value of each divided data set is then calculated [15]. A new time series $\left\{y_{j}^{(\tau)}\right\}$ is constituted; it is expressed as Eq. (6) and Fig. 1.

$$
\left\{y_{j}^{(\tau)}\right\}=\frac{1}{\tau} \sum_{h=(j-1) \tau+1}^{j \tau} x_{h}, \quad 1 \leq j \leq \frac{H}{\tau} .
$$

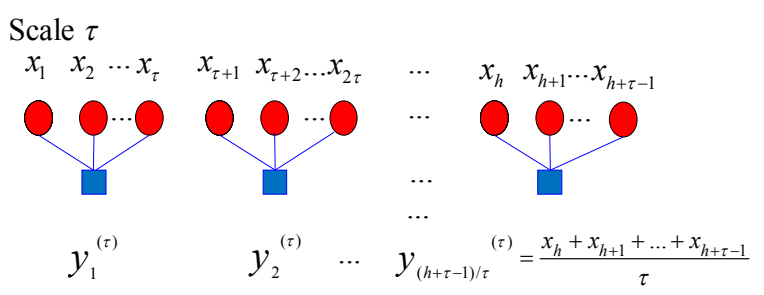

Fig. 1. Multi-scale process

\subsubsection{Fractal Box Dimension}

The fractal box dimension can be used to extract and quantify the feature information included in the signals with different scales. Assuming $X$ is a not empty bounded subsets of $R^{n}$, and $N(X, \varepsilon)$ represents the minimum number of the subset that can cover $X$ set with the maximum diameter $\varepsilon$. The definition of fractal box dimension is expressed as follows [16]:

$$
\operatorname{dim}_{B}(X)=\lim _{\varepsilon \rightarrow 0} \frac{\ln N(X, \varepsilon)}{\ln (1 / \varepsilon)} .
$$

Assuming the signal $Y=\left\{y_{1}, y_{2}, \ldots, y_{Q}\right\} \subset O, O$ is a closed set in the $n$-dimension Euclid space. Because the limitation of Eq. (7) cannot be calculated in accordance with the definition, the approximate method is used. $\varepsilon$ is defined as a baseline, and it is gradually enlarged to $k \varepsilon, k \in Z^{+}$. The calculation equation is as follows:

$$
P(k \varepsilon)=\sum_{i=1}^{Q / k}\left|\begin{array}{l}
\max \left(y_{k(i-1)+1}, y_{k(i-1)+2}, \ldots, y_{k(i-1)+k+1}\right) \\
-\min \left(y_{k(i-1)+1}, y_{k(i-1)+2}, \ldots, y_{k(i-1)+k+1}\right)
\end{array}\right|,
$$

where $i=1,2, \ldots, Q / N ; N$ is the sampling data number, $k=1,2, \ldots, M ; M \leq N$.

The grid count is as follows:

$$
N_{k \varepsilon}=P(k \varepsilon) /(k \varepsilon)+1 ; \quad N_{k \varepsilon}>1 .
$$

A range that has a better linear relationship in the figure $\lg k \varepsilon-\lg N_{k \varepsilon}$ is defined as a non-scaling range, and assuming the beginning and ending of this nonscaling range are $k_{1}$ and $k_{2}$, respectively.

$$
\lg N_{k \varepsilon}=a \lg k \varepsilon+b ; \quad k_{1} \leq k \leq k_{2} .
$$

The slope of this line is determined by the least square method, and it is the fractal box dimension and expressed as follows: 
$d_{B}=\hat{a}=-\frac{\left(k_{2}-k_{1}+1\right)\left(\sum \lg k \lg N_{k \varepsilon}-\sum \lg k \sum \lg N_{k \varepsilon}\right)}{\left(k_{2}-k_{1}+1\right) \sum \lg ^{2} k-\left(\sum \lg k\right)^{2}}$.

\subsection{Extreme Learning Machine}

ELM has the advantages of easy parameter selection, fast learning speed and good generalization performance [17]. The structure of ELM is shown in Fig. 2. Where input layer has $J$ neurons, hidden layer has $H$ neurons, and output layer has $K$ neurons [18].

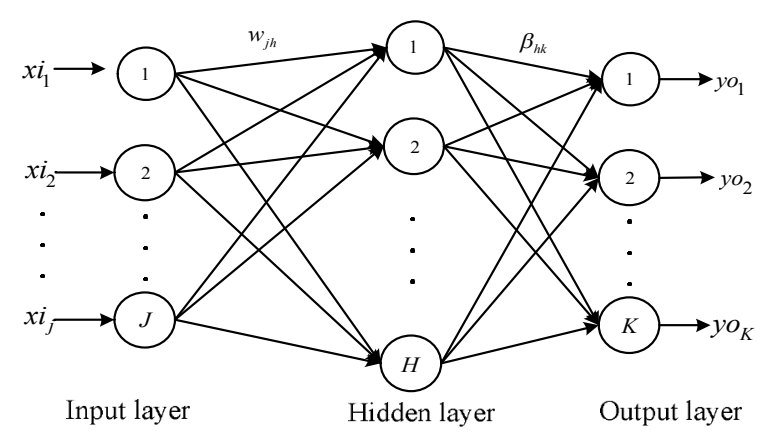

Fig. 2. Structure of $E L M$

The connection weights between the input layer and hidden layer and the connection weights between hidden layer and output layer are expressed as follows:

$$
\begin{aligned}
w & =\left[\begin{array}{cccc}
w_{11} & w_{12} & \ldots & w_{1 h} \\
w_{21} & w_{22} & \ldots & w_{2 h} \\
\vdots & \vdots & \ddots & \vdots \\
w_{j 1} & w_{j 2} & \ldots & w_{j h}
\end{array}\right], \\
\beta & =\left[\begin{array}{cccc}
\beta_{11} & \beta_{12} & \ldots & \beta_{1 k} \\
\beta_{21} & \beta_{22} & \ldots & \beta_{2 k} \\
\vdots & \vdots & \ddots & \vdots \\
\beta_{h 1} & \beta_{h 2} & \ldots & \beta_{h k}
\end{array}\right],
\end{aligned}
$$

where $w_{j h}$ is the connection weight between the $j^{\text {th }}$ neuron of the input layer and the $h^{\text {th }}$ neuron of the hidden layer, and $\beta_{h k}$ is the connection weight between the $h^{\text {th }}$ neuron of the hidden layer and the $k^{\text {th }}$ neuron of the output layer.

Setting the threshold $\phi$ of the hidden neurons is expressed as follows:

$$
\phi=\left[\phi_{1}, \phi_{2}, \ldots, \phi_{h}\right]^{T} .
$$

The input matrix $x i$ and output matrix yo of the training set with $Q$ samples are expressed as follows:

$$
\begin{aligned}
x i & =\left[\begin{array}{cccc}
x i_{11} & x i_{12} & \ldots & x i_{1 Q} \\
x i_{21} & x i_{22} & \ldots & x i_{2 Q} \\
\vdots & \vdots & \ddots & \vdots \\
x i_{j 1} & x i_{j 2} & \ldots & x i_{j Q}
\end{array}\right]_{J \times Q}, \\
y o & =\left[\begin{array}{cccc}
y o_{11} & y o_{12} & \ldots & y o_{1 Q} \\
y o_{21} & y o_{22} & \ldots & y o_{2 Q} \\
\vdots & \vdots & \ddots & \vdots \\
y o_{k 1} & y o_{k 2} & \ldots & y o_{k Q}
\end{array}\right]_{K \times Q} .
\end{aligned}
$$

The activation function of the hidden neuron is $g(x)$, then, the output $T$ of ELM is expressed as follows:

$$
\begin{gathered}
T=\left[t_{1}, t_{2}, \ldots, t_{Q}\right]_{K \times Q}, \\
t_{h}=\left[t_{1 h}, t_{2 h}, \ldots, t_{K h}\right]_{1 \times K}^{T}=\left[\begin{array}{c}
\sum_{i=1}^{H} \beta_{i 1} g\left(w_{i} x i_{h}+\phi_{i}\right) \\
\sum_{i=1}^{H} \beta_{i 2} g\left(w_{i} x i_{h}+\phi_{i}\right) \\
\vdots \\
\sum_{i=1}^{H} \beta_{i K} g\left(w_{i} x i_{h}+\phi_{i}\right)
\end{array}\right]_{K \times 1},
\end{gathered}
$$

where $w_{i}=\left[w_{i 1}, w_{i 2}, \ldots, w_{i J}\right]^{T}, x i_{h}=\left[x i_{1 h}, x i_{2 h}, \ldots, x i_{J h}\right]^{T}$.

The Eq. (16) can be expressed as follows:

$$
H \beta=T^{*},
$$

where $T^{*}$ is the transpose of $T$, and $H$ is the output matrix of the hidden layer of ELM, and its concrete form is expressed as follows:

$$
\begin{aligned}
& H\left(w_{1}, w_{2}, \ldots, w_{H}, \phi_{1}, \phi_{2}, \ldots, \phi_{H}, x i_{1}, x i_{2}, \ldots, x i_{Q}\right)= \\
& =\left[\begin{array}{cccc}
g\left(w_{1} \bullet x i_{1}+\phi_{1}\right) & g\left(w_{2} \bullet x i_{1}+\phi_{2}\right) & \cdots & g\left(w_{h} \bullet x i_{1}+\phi_{h}\right) \\
g\left(w_{1} \bullet x i_{2}+\phi_{1}\right) & g\left(w_{2} \bullet x i_{2}+\phi_{2}\right) & \cdots & g\left(w_{h} \bullet x i_{2}+\phi_{h}\right) \\
\vdots & \vdots & \ddots & \vdots \\
g\left(w_{1} \cdot x i_{Q}+\phi_{1}\right) & g\left(w_{2} \bullet x i_{Q}+\phi_{2}\right) & \cdots & g\left(w_{h} \bullet x i_{Q}+\phi_{h}\right)
\end{array}\right] .
\end{aligned}
$$

When the activation function $g(x)$ is infinitely differentiable, the parameters of ELM do not need to be adjusted. $w$ and $\phi$ can be randomly selected. The connection weight $\beta$ between hidden layer and output layer can be obtained by calculating the least square solution of the following equations [19].

$$
\min _{\beta}=\left\|H \beta-T^{*}\right\| .
$$

The solution of Eq. (19) is as follows:

$$
\hat{\beta}=H^{+} T^{*} \text {, }
$$


where $H^{+}$is the Morre-Penrose generalized inverse matrix of the output matrix $H$.

\section{EXPERIMENT INTRODUCTION}

The planetary gear fault simulation bench is shown in Fig. 3. The planetary gearbox and fixed-axis gearbox are commonly used transmission components, and they have their respective advantages. Due to the need for some special occasions, the planetary gearbox and fixed-axis gearbox are often used in combination, such as the transmission system of shearer cutting part, the transmission system of the wind turbine and automotive gear reducer, etc. Thus, the fault simulation bench combining a planetary gearbox and fixed-axis gearbox is used. The acceleration sensors that used to measure the vibration signal generated by planetary gear are installed on the shell of the

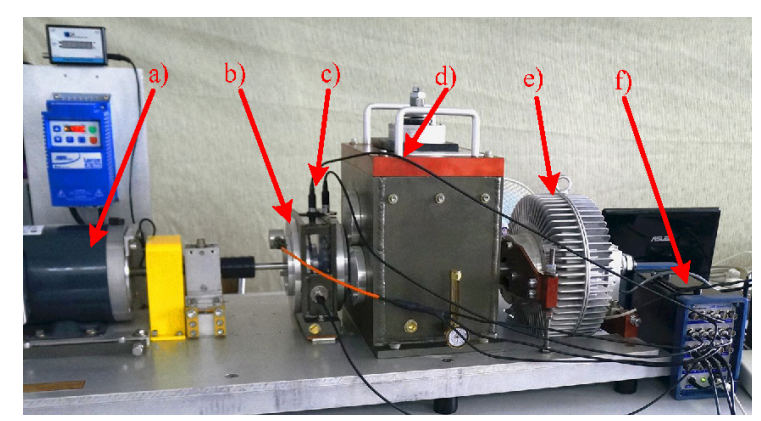

Fig. 3. Fault experiment for planetary gear, a) three-phase asynchronous motor, b) planetary gearbox, c) acceleration sensors, d) fixed-axis gearbox, e) load system, f) acquisition system

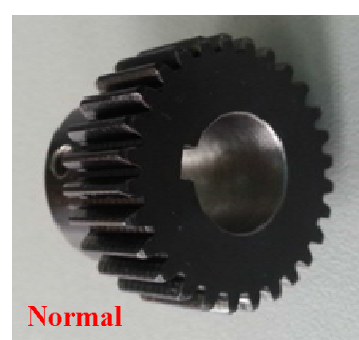

a)

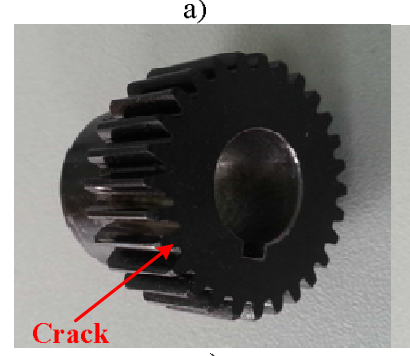

c)

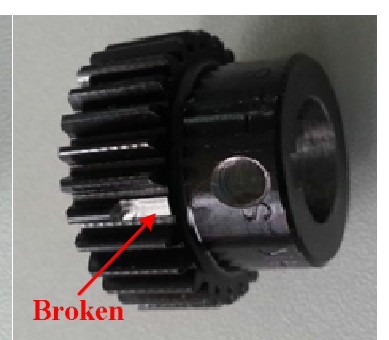

b)

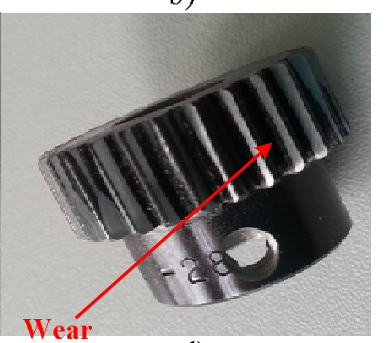

d)
Fig. 4. Four types of gears, a) normal gear, b) broken gear, c) gear with tooth root crack, d) wear gear planetary gearbox. In this experiment, the sun gear faults are simulated; they are normal gear, broken gear, gear with tooth root crack and wear gear. Those gears are shown in Fig. 4. The parameter setting of the experiment process is shown in Table 1, and the vibration signals of four types of planetary gear statuses are sampled. The collected vibration signal is divided into a number of samples for analysis, and each sample includes 8400 data points. They are used to verify the effectiveness of the proposed fault diagnosis method of the planetary gear.

Table 1. Parameters setting of the experiment process

\begin{tabular}{ccc}
\hline Motor speed & Sampling frequency & Load \\
\hline $2400 \mathrm{r} / \mathrm{min}$ & $4200 \mathrm{~Hz}$ & $13.5 \mathrm{Nm}$ \\
\hline
\end{tabular}

\section{EXPERIMENT ANALYSIS}

The analysis flowchart of the fault diagnosis method based on the multi-scale fractal box dimension of CEEMD and ELM is proposed and shown in Fig. 5.

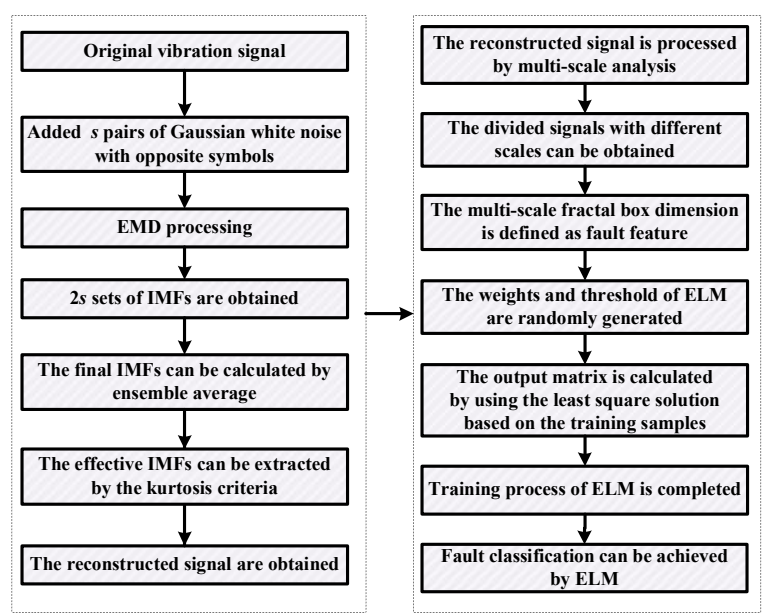

Fig. 5. Experimental analysis flowchart

The vibration signals of four types of planetary gears are collected and shown in Fig. 6. It can be seen that the vibration signals have FM and AM characteristics, and that is because of the influences of complex structure, assembly error, motor, and load. The vibration signal of normal gear includes more shock components, and there are no obvious rules. The gear surface structure and its stiffness are changed when the planetary gear fault occurs, and the additional shock components are generated in the meshing process. Those cause the vibration signals of the faulty gear to be more complex. It can be seen 
from Fig. 6 that the gear status cannot be recognized based on the time-domain signals.

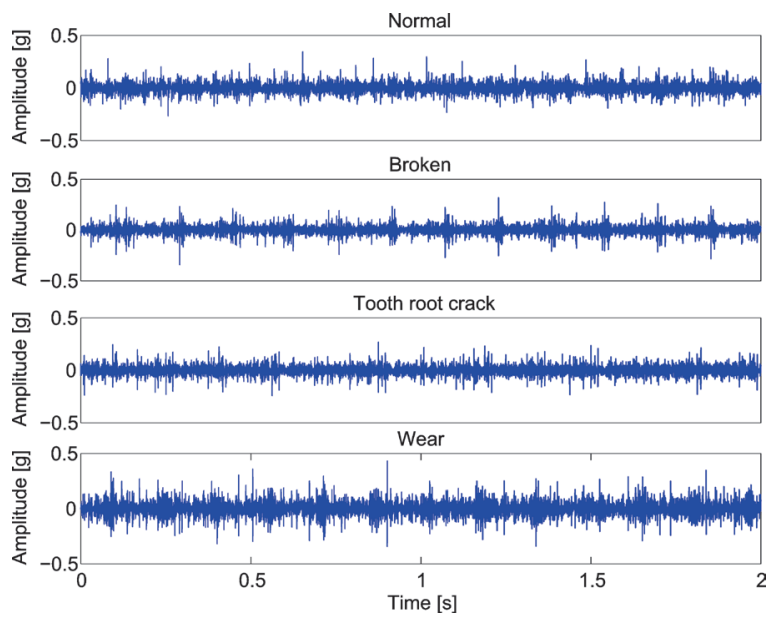

Fig. 6. Four types of vibration signals

The proposed fault diagnosis method is used to process the vibration signal. The vibration signal is sampled as a digital signal, which is composed of a series of data points. It is decomposed by CEEMD according to Eqs. (1) to (4). The number of the added Gaussian white noise $S$ is set to 50 , the amplitude of the added Gaussian white noise $n_{s}(t)$ is set to 0.25 times the standard deviation of the original signal, and EMD process $E()$ is defined in [4]. The vibration signal of wear gear is selected as an example to show the decomposition result of CEEMD, and the decomposition result of EEMD is compared. The decomposition results of EEMD and CEEMD for wear gear are shown in Figs. 7 and 8, respectively.

The complex signal can be expressed as multiple signals with simple characteristics and are easy to analyse with CEEMD; 13 IMFs and a residual signal is obtained, and the residual signal is called IMF14 for convenience. They are arranged from highfrequency to low-frequency. It can be found that the decomposition result of CEEMD is superior to that of EEMD. For EEMD, it is obvious that there is the phenomenon of modal aliasing in IMF6, IMF7, and IMF8. Modal aliasing refers to an IMF that contains the features with great differences or similar features distributed in different IMFs. That causes the waveforms of adjacent IMFs exhibit an aliasing phenomenon, which affects the extraction of the fault features. The quality of the obtained IMFs by CEEMD is greatly improved, and the modal aliasing degree is further reduced. The decomposition errors of EEMD and CEEMD are calculated and shown in Fig. 9. The magnitude of the decomposition error of EEMD is

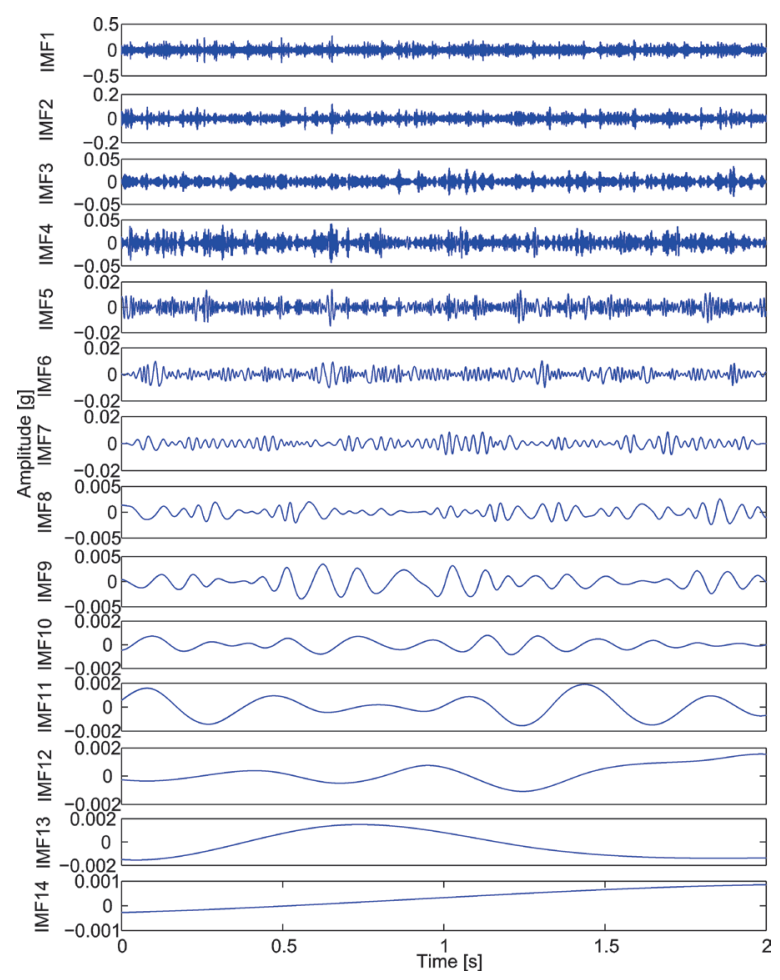

Fig. 7. Decomposition results of EEMD for wear gear

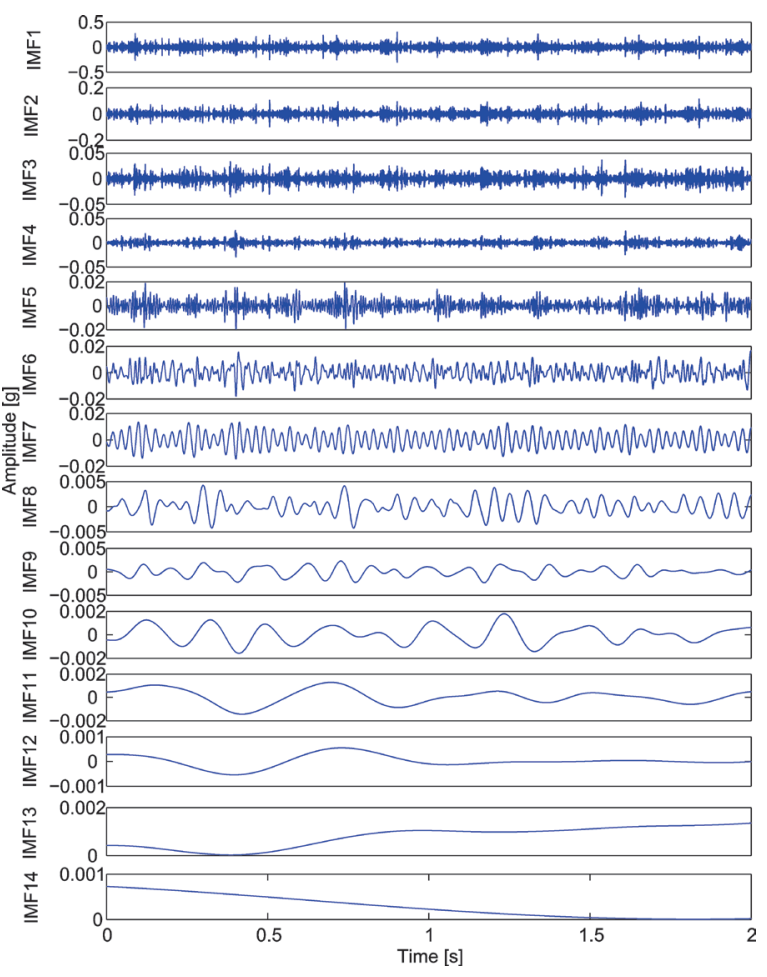

Fig 8. Decomposition result of CEEMD for wear gear

$10^{-4}$, and it is obviously more than that of CEEMD. The magnitude of the decomposition error of CEEMD 
is only $10^{-7}$. CEEMD can better eliminate the residual noise generated by the added Gaussian white noise, and the decomposition error can be reduced.
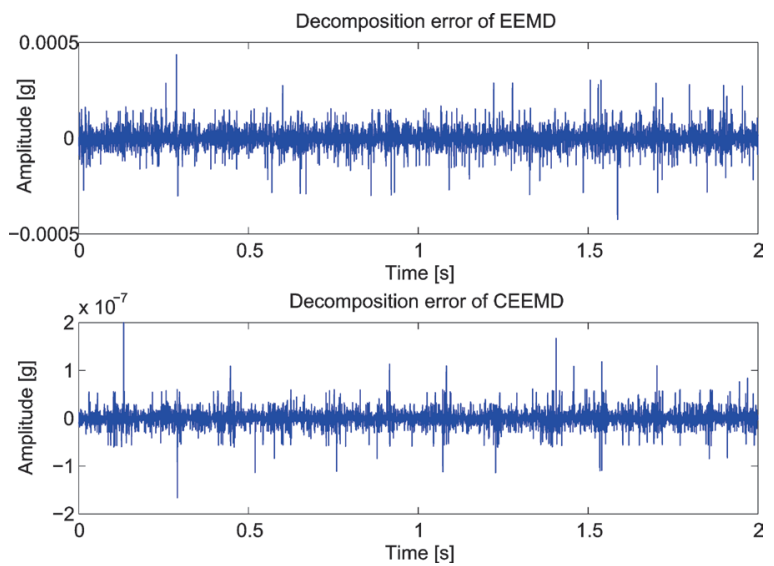

Fig. 9. Decomposition errors of EEMD and CEEMD

The IMFs containing different information can be obtained by CEEMD, and the fault feature information generated by the planetary gear is only contained in some IMFs. The shock components are generated during the meshing process of the planetary gear, and the fault feature information is usually contained in the shock components. Thus, kurtosis is defined as selection criteria to select the effective IMFs associated with fault feature information. Each IMF can be seen as a single digit signal, and the kurtosis of each IMF can be calculated according to Eq. (13), and it is shown in Fig. 10.

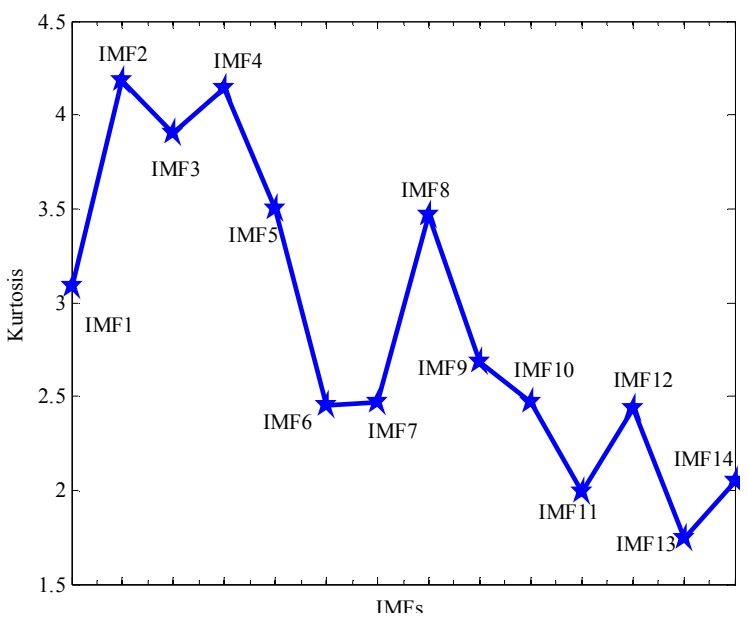

Fig. 10. Kurtosis of each IMF

It can be seen from Fig. 10 that IMF2 has the maximum kurtosis; it reaches 4.1825 . IMF11 has the minimum kurtosis; it reaches 1.9930. In general, more shock components are included in the signal when its kurtosis is greater than 3. Thus, IMF1, IMF2, IMF3, IMF4, IMF5, and IMF8 are selected as the effective IMFs. The reconstructed signal of the effective IMFs can be obtained, and it includes more shock feature information generated by planetary gear faults.

The multi-scale fractal box dimension of the reconstructed signal of the effective IMFs should be extracted. The reconstructed signal includes 8400 data points, and the scale factor $\tau$ is set to 20 . Twenty signals containing different scales are obtained from the reconstructed signal according to Eq. (6), and their fractal box dimensions are calculated according to Eqs. (7) to (11). The multi-scale fractal box dimension of four types of planetary gears is shown in Fig. 11.

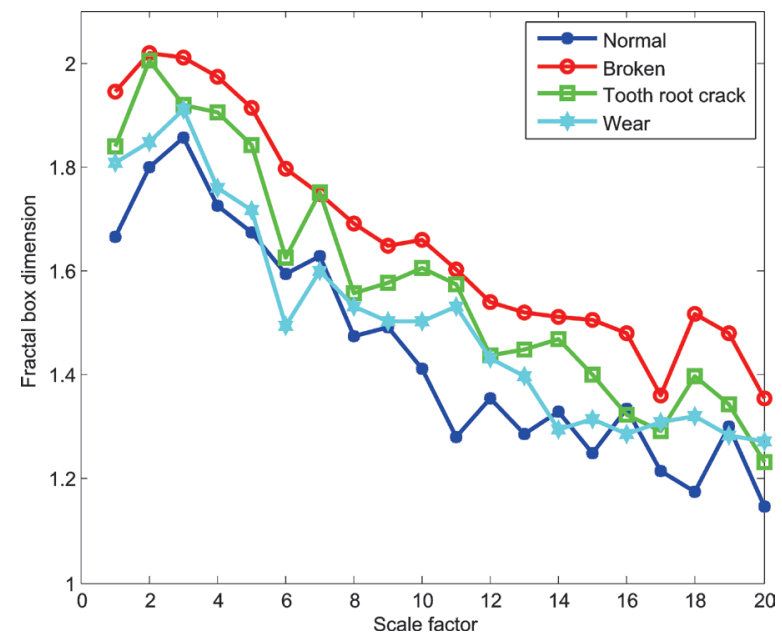

Fig. 11. Multi-scale fractal box dimension of four types of planetary gears

The multi-scale fractal box dimension of four types of planetary gears is decreasing with the increasing of the multi-scale factor $\tau$. Due to the additional shock components being produced when planetary gear faults occur, the multi-scale fractal box dimension of normal gear is less than that of other gears. The fault degree of a broken gear is relatively serious, so its multi-scale fractal box dimension is relatively large. It also can be found that there are the differences of the fractal box dimension under different scales for four types of planetary gears, so the multi-scale fractal box dimension is an effective fault feature. Moreover, next, the planetary gear status can be recognized by combining ELM.

The training sample set is built to train ELM, and each gear status has 50 training samples. The multiscale fractal box dimension of each sample constitutes the input matrix in Eq. (15). Because the multi-scale factor $\tau$ is set to 20, the input matrix has 20 dimensions. Because the samples should be divided into four types, 
the output layer of ELM has four neurons. Meanwhile, four types of gear statuses should be labeled: the normal gear is labeled by [ $\left[\begin{array}{lll}1 & 0 & 0\end{array}\right]$, broken gear is

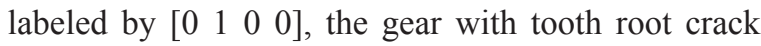
is labeled by $\left[\begin{array}{llll}0 & 0 & 1 & 0\end{array}\right]$, and the wear gear is labeled by $\left[\begin{array}{llll}0 & 0 & 0 & 1\end{array}\right]$, and they constitute the output matrix in Eq. (15). If the number of hidden neurons and the activation function with infinitely differentiable are determined, the connection weight $w$ in Eq. (12) and the threshold $\phi$ of the hidden neurons in Eq. (14) can be randomly selected. The connection weight $\beta$ can be calculated according to Eqs. (16) to (20), and the training process of ELM is completed. The number of hidden neurons and the type of activation function have a large influence on the application of ELM, and in general, the activation function can be selected from 'sig' function, 'sin' function or 'hardlim' function. To determine the optimal number of hidden neurons and the type of activation function, the root mean square error (RMSE) can be used to evaluate the training performance of ELM. The RMSE of the ELM with different hidden neurons and different activation functions is shown in Fig. 12. In addition, in order to verify the recognition performance of ELM, the testing sample set is built. The vibration signals of four types of planetary gear statuses are divided into the testing samples to be analysed, and each gear status has 50 testing samples. The testing samples are recognized by the trained ELM, and the overall recognition rate of the ELM with different hidden neurons and different activation functions is shown in Fig. 13.

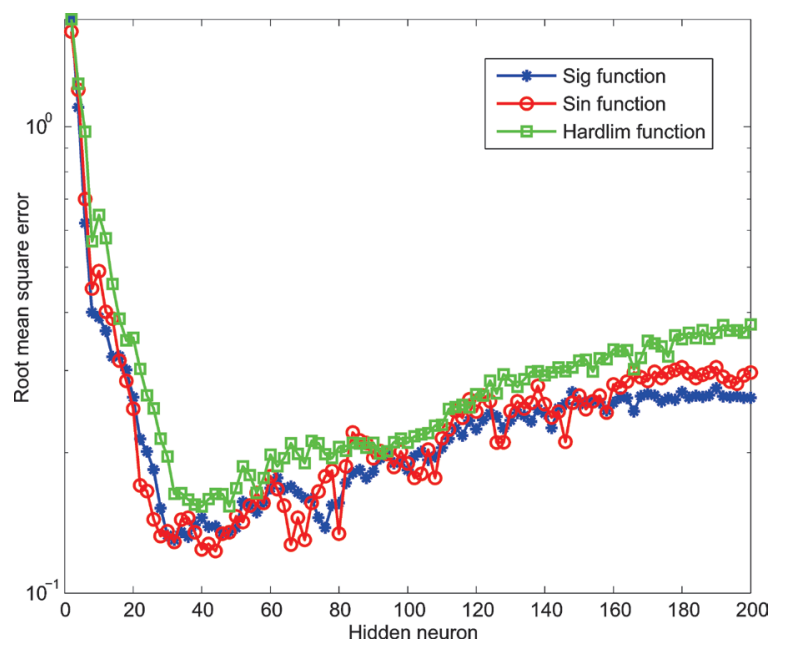

Fig. 12. RMSE of the ELM with different hidden neurons and different activation functions

For different activation functions, it can be found from Fig. 12 that the RMSE has a smaller value when the number of hidden neurons is between 30 and 50. Moreover, when the number of hidden neurons reaches a certain level, the RMSE increases with the increasing of hidden neurons. The ELM with 44 hidden neurons and 'sin' function has the minimum RMSE, and it reaches 0.1230 . In addition, it can be seen from Fig. 13 that different hidden neurons and different activation functions have great influence on the recognition performance of ELM. When the number of hidden neurons increases to a certain extent, the overall recognition rate is gradually decreasing. The overall recognition rate has a maximum value when the ELM has 44 hidden neurons and 'sin' function, and it reaches $92.5 \%$. Therefore, the ELM with 44 hidden neurons and 'sin' function has the best recognition performance, and the detailed recognition rate of the ELM for different planetary gear statuses is shown in Table 2.

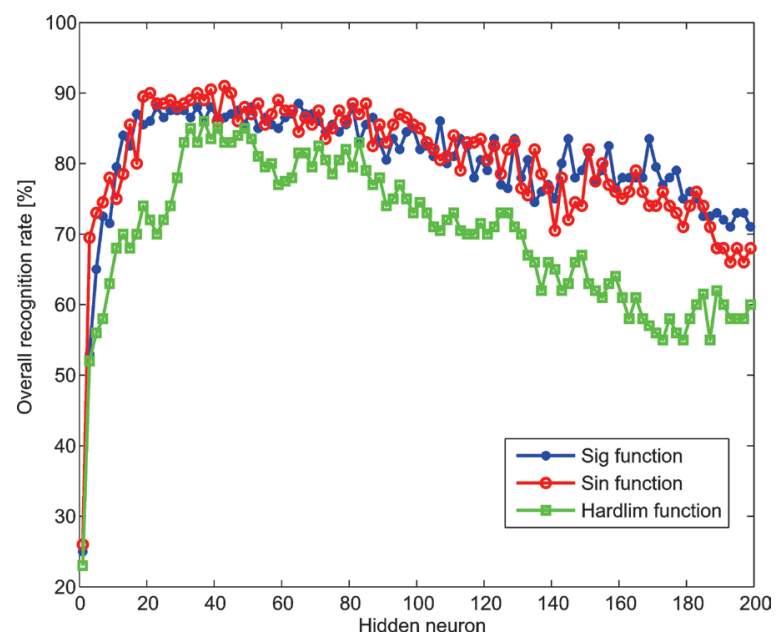

Fig. 13. Overall recognition rate of the ELM with different hidden neurons and different activation functions

Table 2. Detailed recognition rate of the ELM with 44 hidden neurons and 'sin' function

\begin{tabular}{lcccc}
\hline & $\begin{array}{c}\text { Normal } \\
\text { gear }\end{array}$ & $\begin{array}{c}\text { Broken } \\
\text { gear }\end{array}$ & $\begin{array}{c}\text { Tooth root } \\
\text { crack }\end{array}$ & $\begin{array}{c}\text { Wear } \\
\text { gear }\end{array}$ \\
\hline Recognition rate [\%] & 94 & 100 & 86 & 90 \\
\hline
\end{tabular}

The testing samples of four types of planetary gear statuses are used to verify the recognition performance of ELM. The recognition rate of each planetary gear status is obtained by calculating the number correctly recognized by ELM when the testing samples belong to some planetary gear status that is recognized by ELM. The multi-scale fractal box dimension of the broken gear is the largest, and it has a great difference with other gears; therefore, 
the broken gear has the best recognition rate, and it reaches $100 \%$. The recognition rate of gear with tooth root crack is relatively low, and it reaches $86 \%$. The recognition rates of normal gear and wear gear are $94 \%$ and $90 \%$, respectively. The ELM with 44 hidden neurons and 'sin' function has a better recognition rate for different planetary gear statuses. The recognition rate can be used to show the ability to distinguish different planetary gear statuses, and it also can be seen as the credibility of the recognition result of ELM. In the practical application process, the vibration signal is continuously collected, and it also needs to be divided into multiple continuous samples for processing. More samples should be used to improve the reliability of the results, and the final diagnosis result needs to be determined by the recognition results of multiple samples. Next, the proposed method is applied to industrial experiments.

\section{INDUSTRIAL EXPERIMENT APPLICATION}

The fault diagnosis system based on the proposed method is applied to an industrial experiment in the Xutuan coal mine in the Anhui province of China, and it is used to monitor the planetary gear of shearer cutting parts. Before the establishment of the fault diagnosis system, the training samples of different planetary gear statuses of shearer cutting parts are prepared, and their fault features are extracted to train the fault diagnosis system according to the proposed method. A fault diagnosis system can be established, and it is used to identify the planetary gear faults of shearer cutting part. In the practical application process for shearer cutting part, the acceleration sensor is installed on the shell of the bearing seat of the planetary gear, and the data acquisition instrument is used to sample the signal of the acceleration sensor. The collected signal is transmitted to the PC fault diagnosis software, which is compiled with Labview. The experiment is shown in Fig. 14, and the data acquisition instrument is shown in Fig. 15.

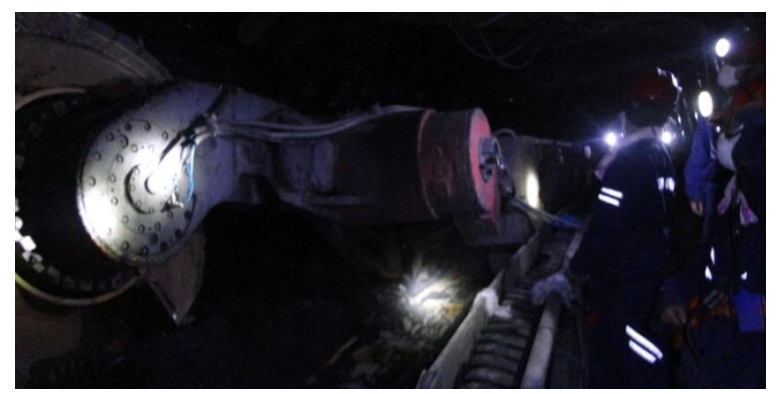

Fig. 14. Diagnosis experiment of planetary gear of shearer cutting part

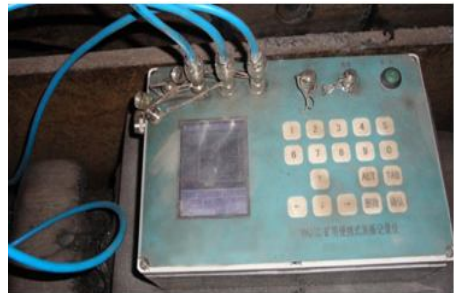

Fig. 15. Data acquisition instrument

The sampling frequency is set to $5000 \mathrm{~Hz}$. Because the vibration signal is collected continuously, it needs to be divided into multiple samples for analysis, and each sample is set to include 10000 data points. In the process of diagnosis analysis, 50 samples are set to be analysed each time, and the diagnosis result of each sample can be obtained. The probabilities belonging to different statuses can be counted. The final diagnosis result is the status with the maximum probability, and the corresponding probability can be regarded as the reliability of the final diagnosis result. More samples can improve the accuracy and reliability of diagnosis. Next, an example of the detected fault in the experiment application is selected to illustrate. The vibration signals of normal operation and the detected fault, which are obtained by the established fault diagnosis system is shown in Fig. 16. In addition, their multi-scale fractal box dimensions are extracted according to the proposed method, and they are shown in Fig. 17.

a)

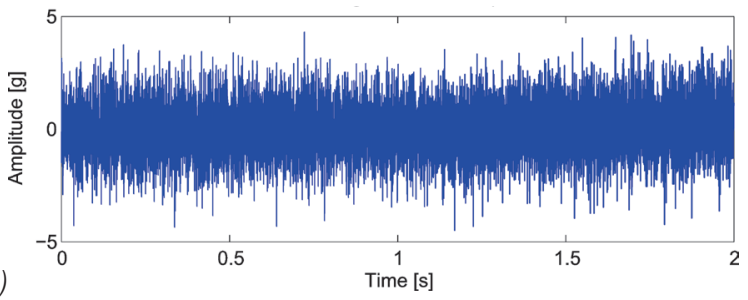

b)

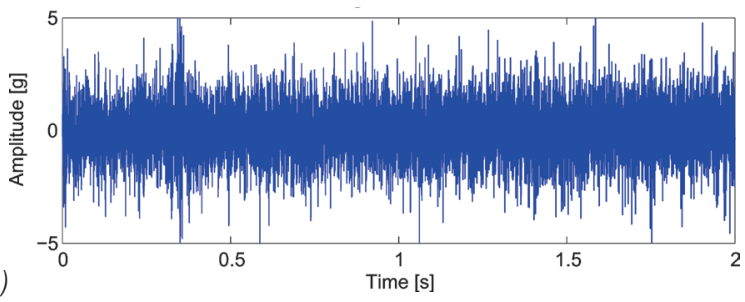

Fig. 16. The vibration signals of a) normal operation, and b) detected fault

It can be seen from the figures that the vibration amplitude of the detected fault is larger in comparison with the vibration signal of normal operation. The shock components are increased, and their amplitude 


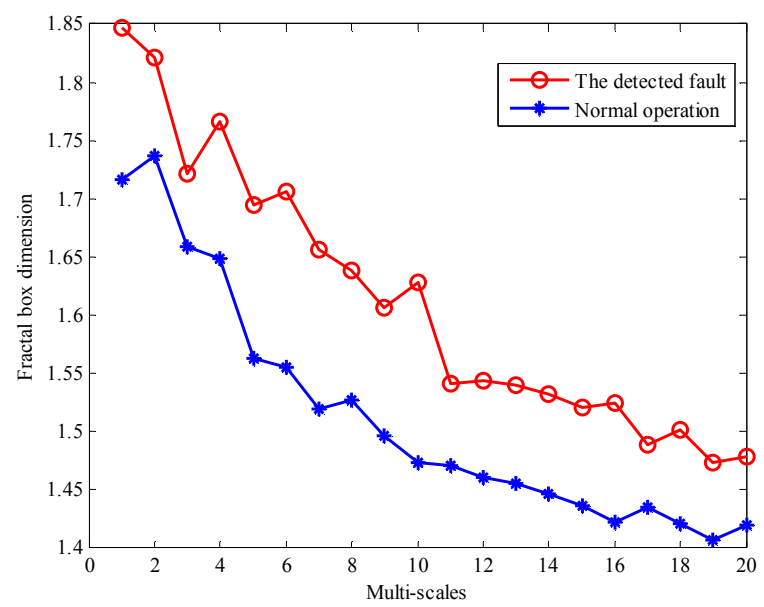

Fig. 17. Multi-scale fractal box dimensions of normal operation and the detected fault

is relatively strong. The multi-scale fractal box dimension of the detected fault increases comparing with that of normal operation, and it greatly deviates from the multi-scale fractal box dimension of normal operations.

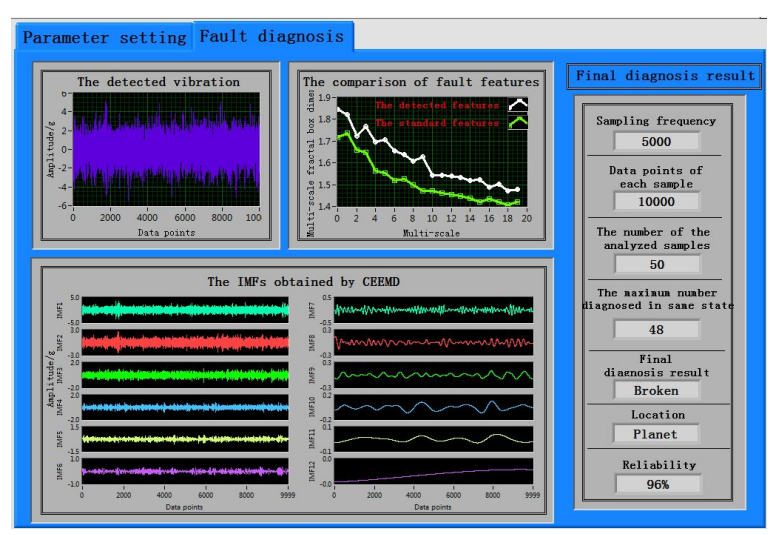

Fig. 18. Fault diagnosis software

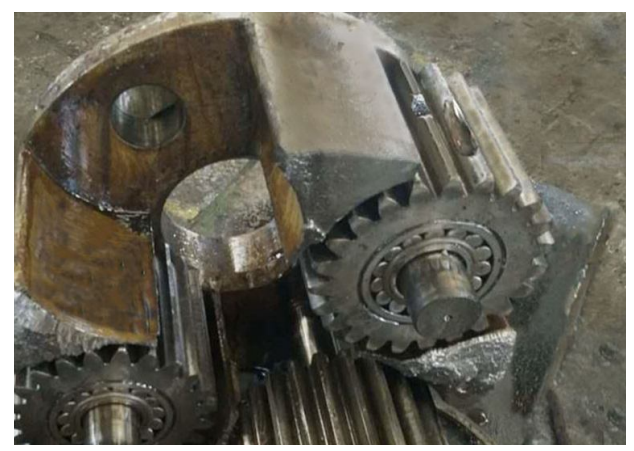

Fig. 19. The diagnosed broken gear

In the established fault diagnosis system, the fault diagnosis software is shown in Fig. 18. The final diagnosis results, the detected vibration signal, the IMFs obtained by CEEMD, the comparison between the detected features and the standard features can be displayed on the software interface. The number of analysis samples is set to 50, and 48 of them are diagnosed as broken gear. Thus, the final diagnosis result is that the monitored planetary gear is broken, and the credibility of the final diagnosis result is $96 \%$. The shearer cutting part is repaired after determining the diagnosis result, and it is found that the breakage happens on the first stage planetary gear of shearer cutting part. The diagnosed broken gear is shown in Fig. 19. The experiment proves that the proposed fault diagnosis method of a planetary gear based on multiscale fractal box dimension of CEEMD and ELM can accurately recognize the planetary gear status, and it is an effective fault diagnosis method.

\section{CONCLUSIONS}

A fault diagnosis method of planetary gear based on multi-scale fractal box dimension of CEEMD and ELM is proposed. CEEMD is proposed to improve the decomposition effect of EEMD, and a series of highquality IMFs which contain more accurate feature information can be obtained by CEEMD. The effective IMFs associated with the shock feature information generated by planetary gear faults are selected by the kurtosis criterion, and they are reconstructed. The reconstructed signal is processed by the multi-scale algorithm, and the feature information contained in the signals with different scales is extracted and quantified by the fractal box dimension. The multiscale fractal box dimension is used as the fault feature information, and it is defined as the input of ELM. The influence of the number of hidden neurons and the type of activation function on the recognition performance of ELM is analysed. The testing sample set is built and used to verify the recognition effect, and the recognition rates of the ELM with different hidden neurons and different activation functions are calculated and compared. In addition, a fault diagnosis system is established based on the proposed method, and the industrial experiment application is carried out. The experiments show that the proposed method based on multi-scale fractal box dimension of CEEMD and ELM can be used to recognize planetary gear status, and it is an effective fault diagnosis method for planetary gears. 


\section{ACKNOWLEDGEMENTS}

This work was supported by a Project Funded by the Priority Academic Program Development of Jiangsu Higher Education Institutions, the Topnotch Academic Programs Project of Jiangsu Higher Education Institutions, Natural Science Foundation of Jiangsu Province (Grant Number BK20141128) and Fundamental Research Funds for the Central Universities (grant number 2015YC02); this support is gratefully acknowledged.

\section{REFERENCES}

[1] Chen, X.H., Cheng, G., Shan, X.L., Hu, X., Guo, Q. (2015). Research of weak fault feature information extraction of planetary gear based on ensemble empirical mode decomposition and adaptive stochastic resonance. Measurement, vol. 73, p. 55-67, D0l:10.1016/j. measurement.2015.05.007.

[2] Fyler, D.C., Inalpolat, M. (2016). A dynamic model for doubleplanet planetary gearsets. Journal of Vibration and Acoustics, vol. 138, no. 2, Article ID 021006, D0I:10.1115/1.4032181.

[3] Do, V.T., Nguyen, L.C. (2016). Adaptive empirical mode decomposition for bearing fault detection. Strojniški vestnik Journal of Mechanical Engineering, vol. 62, no. 5, p. 281-290, DOI:10.5545/sv-jme.2015.3079.

[4] Huang, N.E., Shen, Z., Long, S.R. (1998). The empirical mode decomposition and the Hilbert spectrum for nonlinear and non-stationary time series analysis. Proceedings of the Royal Society A, vol. 454, p. 903-995, Dol:10.1098/rspa.1998.0193.

[5] Cheng, G., Cheng, Y.L., Shen, L.H., Qiu J.B., Zhang, S. (2013). Gear fault identification based on Hilbert-Huang transform and SOM neural network. Measurement, vol. 46, no. 3, p. 1137-1146, Dol: 10.1016/j.measurement.2012.10.026.

[6] Wu, Z., Huang, N.E. (2009). Ensemble empirical mode decomposition: a noise-assisted data analysis method. Advances in Adaptive Data Analysis, vol. 1, no. 1, p. 1-41, DOI:10.1142/S1793536909000047.

[7] Perez-Ramirez, C.A., Amezquita-Sanchez, J.P., Adeli, H., Valtierra-Rodriguez, M., Camarena-Martinez, D., J. Romero-Troncoso, R.J. (2016). New methodology for modal parameters identification of smart civil structures using ambient vibrations and synchrosqueezed wavelet transform. Engineering Applications of Artificial Intelligence, vol. 48, p. 1-12, Dol:10.1016/j.engappai.2015.10.005.

[8] Begum, S., Barua, S., Filla, R., Ahmed, M.U. (2014). Classification of physiological signals for wheel loader operators using multi-scale entropy analysis and case-based reasoning. Expert Systems with Applications, vol. 41, no. 2, p. 295-305, Dol:10.1016/j.eswa.2013.05.068.
[9] Liu, Y., Lin, J.L., Chen, K. (2015). A stable algorithm of box fractal dimension and its application in pore structure. Rare Metal Materials and Engineering, vol. 44, no. 4, p. 800-804, Dol:10.1016/S1875-5372(15)30050-3.

[10] Khazaee, M., Ahmadi, H., Omid, M. (2013). Feature-level fusion based on wavelet transform and artificial neural network for fault diagnosis of planetary gearbox using acoustic and vibration signals. Insight, vol. 55, no. 6, p. 323-329, Dol:10.1784/insi.2012.55.6.323.

[11] Aghbashlo, M., Shamshirband, S., Tabatabaeic, M., Yee, P.L., Larimi, Y.N. (2016). The use of ELM-WT (extreme learning machine with wavelet transform algorithm) to predict exergetic performance of a DI diesel engine running on diesel/biodiesel blends containing polymer waste. Energy, vol. 94, p. 443-456, D0l:10.1016/j.energy.2015.11.008.

[12] Ahila, R., Sadasivam, V., Manimala, K. (2015). An integrated PSO for parameter determination and feature selection of ELM and its application in classification of power system disturbances. Applied Soft Computing, vol. 32, p. 23-37, DOI:10.1016/j.asoc.2015.03.036.

[13] Colominas, M.A., Schlotthauer, G., Torres, M.E. (2014). Improved complete ensemble EMD: A suitable tool for biomedical signal processing. Biomedical Signal Processing and Control, vol. 14, p. 19-29, D0l:10.1016/j. bspc.2014.06.009.

[14] Cong, F.Y., Chen, J., Dong, G.M. (2012). Spectral kurtosis based on AR model for fault diagnosis and condition monitoring of rolling bearing. Journal of Mechanical Science and Technology, vol. 26, no. 2, p. 301-306, D0l:10.1007/ s12206-011-1029-0.

[15] Li, Y.B., Xu, M.L., Wang, R.X., Huang, W.H. (2016). A fault diagnosis scheme for rolling bearing based on local mean decomposition and improved multiscale fuzzy entropy. Journal of Sound and Vibration, vol. 360, p. 277-299, D0l:10.1016/j. jsv.2015.09.016.

[16] Zheng, Z., Jiang, W., Wang, Z., Zhu, Y., Yang, K. (2015). Gear fault diagnosis method based on local mean decomposition and generalized morphological fractal dimensions. Mechanism and Machine Theory, vol. 91, p. 151-167, D0I:10.1016/j.mechmachtheory.2015.04.009.

[17] Sá, J.J.M., Backes, A.R. (2016). ELM based signature for texture classification. Pattern Recognition, vol. 51, p. 395401, D0I:10.1016/j.patcog.2015.09.014.

[18] Heras, D.B., Argüello, F., Quesada-Barriuso, P. (2014). Exploring ELM-based spatial-spectral classification of hyperspectral images. International Journal of Remote Sensing, vol. 35, no. 2, p. 401-423, D0I:10.1080/01431161.2013.869633.

[19] Huang, G.B., Zhou, H., Ding, X., Zhang, R. (2012). Extreme learning machine for regression and multiclass classification. IEEE Transactions on Systems, Man, and Cybernetics, Part B: Cybernetics, vol. 42, no. 2, p. 513-529, Dol:10.1109/ TSMCB.2011.2168604. 